\title{
SHEAF THEORETIC COHOMOLOGICAL DIMENSION AND FINITISTIC SPACES
}

\author{
SATYA DEO
}

\begin{abstract}
For a topological $n$-manifold $X$, we proved earlier [7] that $\operatorname{Dim}_{\mathbf{z}}(X)=n$ +1 , if $n>0$; and, for a zero-dimensional manifold (discrete space) we observed that $\operatorname{Dim}_{\mathbf{z}}(X)=0$. The question was later raised as to what are those paracompact spaces, besides discrete one, for which $\operatorname{Dim}_{\mathbf{z}}(X)=0$. In this paper we prove that there is none, i.e., if $X$ is not discrete then $\operatorname{Dim}_{\mathbf{z}}(X)>0$. Another question which cropped up only recently in the cohomological theory of topological transformation groups is whether or not there exists a finitistic space which is not of finite (sheaf theoretic) integral cohomological dimension. We show that this question is related to a famous unsolved problem of cohomological dimension theory.
\end{abstract}

1. Introduction and preliminaries. Unless stated otherwise, all our spaces are assumed to be completely regular Hausdorff. Let $X$ be a topological space and $L$ be a ring. Suppose $\varphi$ is a family of supports on $X$, and for any sheaf $Q$ of $L$-modules on $X, H_{\varphi}^{*}(X ; \mathbb{Q})$ denotes the Grothendieck cohomology groups of $\mathbb{Q}$ with supports in $\varphi$. Then the largest integer $n($ or $\infty$ ) for which there exists a sheaf $\mathcal{Q}$ of $L$-modules on $X$ such that $H_{\varphi}^{n}(X ; \mathbb{Q}) \neq 0$, is called the cohomological $\varphi$-dimension of $X$ over $L$ and is denoted by $\operatorname{dim}_{\varphi, L}(X)$. Furthermore, $\sup _{\varphi}\left\{\operatorname{dim}_{\varphi, L}(X)\right\}$ is called the large cohomological dimension of $X$ over $L$ and is denoted by $\operatorname{Dim}_{L}(X)$. Recall that the extent $E(\varphi)$ of a family of supports is the union of all members of $\varphi$. If $\varphi$ is a paracompactifying family of supports on $X$ such that $E(\varphi)=X$, then $\operatorname{dim}_{\varphi, L}(X)$ turns out to be independent of $\varphi[2]$; it is called the cohomological dimension of $X$ over $L$ and is denoted by $\operatorname{dim}_{L}(X)$. In [7] we proved that if $X$ is an $n$-manifold, $n>0$, then $\operatorname{Dim}_{\mathbf{z}}(X)=\operatorname{dim} X+1$, where $\operatorname{dim} X$ denotes the covering dimension of $X$ and agrees with $\operatorname{dim}_{\mathbf{z}}(X)$ whenever the former is finite. We also remarked there [7] that if $X$ is a zero-dimensional manifold (discrete space), then $\operatorname{Dim}_{\mathbf{z}}(X)=0$. It is easy to observe that there are large number of zero-dimensional spaces, other than discrete spaces, for which $\operatorname{Dim}_{\mathbf{z}}(X) \neq 0$. Therefore, it is natural to ask whether or not there is a space $X$ which is not discrete and for which $\operatorname{Dim}_{\mathbf{z}}(X)=0$. We prove that there is none, i.e., for a locally paracompact space $X, \operatorname{Dim}_{\mathbf{Z}}(X)=0$ if and only if $X$ is discrete (Theorem 2.4). Obviously 'if part' is trivial. Our proof of the 'only if' part is not difficult if the space $X$ is either locally compact or first countable. However, our general proof for any locally paracompact space depends on an assumption viz., there does not exist a strongly inaccessible cardinal number. As is well

Received by the editors May 15, 1981.

1980 Mathematics Subject Classification. Primary 55N30; Secondary 54F45.

Key words and phrases. Sheaves, family of supports, sheaf theoretic cohomological dimension, realcompactness, finitistic spaces. 
known, a discrete space is realcompact iff its cardinal number is nonmeasurable [10, p. 163]; and for all practical purposes almost all cardinal numbers are nonmeasurable,i.e., there does not exist a strongly inaccessible cardinal number. Therefore this assumption practically does not amount to any condition for the validity of our proof.

Another question that we discuss in this paper is regarding finitistic spaces. Recall that a paracompact Hausdorff space $X$ is said to be finitistic if each open cover of $X$ has a finite dimensional open refinement $[3,9]$. In the cohomological study of various generalizations of Smith fixed-point theorems and orbit structures of a transformation group $(G, X)$ where $G$ is a compact Lie group, the basic assumption on the space $X$ is any one of the following:

(a) $X$ is compact Hausdorff,

(b) $X$ is paracompact Hausdorff of finite cohomological dimension over $\mathbf{Z}$ or a prime field,

(c) $X$ is finitistic.

Most of the fundamental results $[4,11]$ of cohomological theory of topological transformation groups, specially various generalizations of Smith fixed-point theorems, were earlier obtained for the class of spaces mentioned in (a) or (b). However, in proving the same results for Čech cohomology, it is the class of finitistic spaces, discovered by Swan [15], which fits very well. The trend of proving results for finitistic spaces is now firmly established $[3,9]$. Obviously each compact space is finitistic, but the following appears to be an open

Problem A. Does there exist a paracompact Hausdorff space of finite integral cohomological dimension which is not finitistic?

We do not know an answer to this problem. However, we show that this question is related to a famous unsolved Alexandroff's problem, viz., does there exist a compact Hausdorff space of infinite covering dimension which has finite cohomological dimension with respect to each coefficient group?

All definitions regarding sheaf cohomology, dimension theory and group actions are standard ones.

2. Large zero-dimensional spaces are discrete. Our proof of the main result (Theorem 2.4) of this section will make use of the following characterization of $\operatorname{Dim}_{\mathbf{z}}(X)$ [7]: $\operatorname{Dim}_{\mathbf{z}}(X) \leqslant 0$ if and only if every sheaf of abelian groups on $X$ is flabby. Thus we wish to characterize those completely regular spaces on which every sheaf of abelian groups is flabby; and what we claim is that discrete spaces are the only ones on which every sheaf of abelian groups is flabby. To prove this result first we have the following lemmas.

Lemma 2.1. Suppose $X$ is a locally compact Hausdorff space which is not discrete. Then there exists a countable discrete subset $A$ of $X$ which is not closed.

Proof. One can assume, without loss of generality, that $X$ is compact Hausdorff. By the Bolzano-Weierstrass property, it is then enough to show that there exists a countably infinite discrete subset $A$ of $X$. Since $X$ is not discrete there exists a point $x_{0} \in X$ which is not isolated. Hence, there exists a net $\left(x_{\lambda}\right)$ in $X-\left\{x_{0}\right\}$ which 
converges to $x_{0}$. Let $x_{\lambda_{0}}$ be some element of the net. Use complete regularity of $X$ to find an open niehgbourhood $U_{1}$ of $x_{0}$ such that $x_{\lambda_{0}} \notin \bar{U}_{1}$. Next choose a point $x_{\lambda_{1}}$ of the net so that $\lambda_{1}>\lambda_{0}$ and $x_{\lambda_{1}} \in U_{1}$. Again find an open neighbourhood $U_{2}$ of $x_{0}$ such that $\bar{U}_{2} \subseteq U_{1}-\left\{x_{\lambda_{1}}\right\}$. Proceeding inductively, choose open neighbourhoods $U_{n}$ of $x_{0}$ and points $x_{\lambda_{n}}$ of the net so that for each $n$

$$
\lambda_{n+1}>\lambda_{n}, \quad x_{\lambda_{n}} \in U_{n}, \quad \bar{U}_{n+1} \subseteq U_{n}-\left\{x_{\lambda_{n}}\right\} .
$$

Then, clearly $A=\left\{x_{\lambda_{n}} \mid n \in \mathbf{N}\right\}$ is a countable discrete subset of $X$.

By an obvious modification of the above proof one can easily prove the following

LEMMA 2.2. Let $X$ be a first countable space (e.g. metric space) which is not discrete. Then there exists a countable discrete subset $A$ of $X$ which is not closed.

Lemma 2.3. Let $X$ be a zero-dimensional completely regular space which is not discrete. Then there exists a discrete subset $A$ of $X$ which is not closed.

Proof. Suppose $x_{0} \in X$ is not isolated. Since $X$ is zero-dimensional, $x_{0}$ has a local base of closed-open neighbourhoods of $x_{0}$ [10, p. 247]. Let $V_{1}=X$ and well order the set $X-\left\{x_{0}\right\}=\left\{x_{\alpha} \mid \alpha<\tau\right\}$. For each positive integer $n>1$, choose a closedopen neighbourhood $V_{n}$ of $x_{0}$ such that

$$
V_{1} \supsetneqq V_{2} \supsetneqq \cdots \supsetneqq V_{n} \supsetneqq \cdots
$$

Then $\left\{V_{n}-V_{n+1} \mid n=1,2, \ldots\right\}$ is a disjoint family of nonempty closed-open subsets of $X$. If the union $M$ of this family is not closed, then there exists a net $\left(x_{\lambda}\right)$ in $M$ which converges to some point $x \in X-M$. Because each member $V_{n}-V_{n+1}$ of the family is closed in $M$, the net cannot be frequently in $V_{n}-V_{n+1}$; and also it has to intersect the family $\left\{V_{n}-V_{n+1}\right\}$ in a cofinal subfamily. Then, it is easy to see that one can find a subset $\left(x_{\varphi(\lambda)}\right)$ of $\left(x_{\lambda}\right)$ which has the property that if it intersects $V_{n}-V_{n+1}$, it intersects only once. Then the subset $A=\left\{x_{\varphi(\lambda)}\right\}$ of $X$ is discrete and its closure must contain the point $x \in X-A$, i.e., $A$ is not closed. However, if $M$ is closed then $\bigcap_{n<\omega} V_{n}$ is closed-open and contains infinitely many points of $X$ other than $x_{0}$. Again we continue, by transfinite induction, to find closed-open neighbourhoods $V_{\omega+n}$ of $x_{0}$ such that

$$
\bigcap_{n<\omega} V_{n} \supsetneqq V_{\omega} \supsetneqq V_{\omega+1} \supsetneqq \cdots \supsetneqq V_{\omega+n} \supsetneqq \cdots .
$$

Once again, if the union $M$ of the family $\left\{V_{\alpha}-V_{\alpha+1} \mid \alpha<2 \omega\right\}$ is not closed, we can find, as above, a discrete subset $A$ of $X$ which is not closed; and if $M$ is closed we again continue as above. It is, however, obvious that there must exist a limit ordinal $\tau_{0} \leqslant \tau$ such that

$$
\bigcap\left\{V_{\alpha} \mid \alpha<\tau_{0}\right\}=\left\{x_{0}\right\}
$$

Therefore, the set $\cup\left\{V_{\alpha}-V_{\alpha+1} \mid \alpha<\tau_{0}\right\}=X-\left\{x_{0}\right\}$ cannot be closed and consequently we can find, as above, a discrete subset $A$ of $X$ which is not closed.

REMARK. Although Lemmas 2.1 and 2.2 are special cases of Lemma 2.3, the proof of Lemma 2.1 has been included only to point out that if the space is either locally 
compact Hausdorff or metric then the discrete set $A$, which is not closed, can be chosen to be countable.

We recall that $\operatorname{dim}_{\mathbf{z}}(X)$ is not always defined unless the space $X$ admits a paracompactifying family of supports whose extent is $X$. But, if $X$ is locally paracompact then it always admits a paracompactifying family of supports whose extent equals $X$. Hence $\operatorname{dim}_{\mathbf{z}}(X)$ is always defined if $X$ is locally paracompact. On the other hand, $\operatorname{Dim}_{\mathbf{z}}(X)$ is always defined for any space $X$. However, it is only the class of locally paracompact spaces (which includes locally compact and paracompact spaces) for which sheaf theoretic cohomological dimension is useful in the cohomological theory of topological transformation groups. That is why we prove the following theorem for locally paracompact spaces, although the result will remain true if $X$ is any completely regular space. Note that a locally paracompact space is completely regular. Now we have

TheORem 2.4. Let $X$ be a locally paracompact space which is not discrete. Then $\operatorname{Dim}_{\mathbf{z}}(X)>0$.

Proof. Since each point of $X$ has a closed paracompact neighbourhood and there is a nonisolated point in $X$, there must be a closed paracompact subset $Y$ of $X$ which is not discrete. Because $\operatorname{Dim}_{\mathbf{z}}$ satisfies the monotonicity condition for locally closed subsets, it suffices to show that $\operatorname{Dim}_{\mathbf{Z}} Y>0$. Suppose $\operatorname{dim} Y>0$. Then $Y$ being paracompact $\operatorname{dim}_{\mathrm{Z}} Y=\operatorname{dim} Y$ [12], and consequently

$$
\operatorname{Dim}_{\mathbf{Z}}(Y) \geqslant \operatorname{dim}_{\mathbf{Z}}(Y)>0 \text {. }
$$

Hence, we can assume that $\operatorname{dim} Y=0$. Once again, it suffices to show that there is a closed subset $F$ of $Y$ for which $\operatorname{Dim}_{\mathbf{z}}(F)>0$. Now, by Lemma 2.3, there exists a discrete subset $A$ of $Y$ which is not closed. This means $A$ is open in $\bar{A}$ (closure of $A$ ). Assuming that there does not exist a strongly inaccessible cardinal, we find that $A$ is realcompact. Since $A$ is dense in $\bar{A}$ and $A \neq \bar{A}, A$ cannot be $C$-embedded in $\bar{A}$ [10, p. 125, Example 8A.1]. Suppose $f: A \rightarrow \mathbf{R}$ is a continuous function which does not extend to $\bar{A}$. Consider the constant sheaf $G=\bar{A} \times \mathbf{R}$ of abelian groups on $\bar{A}$. Then, $a \mapsto(a, f(a))$ defines a continuous section $s$ of $G$ on the open set $A$ of $\bar{A}$. Since $f$ cannot be extended continuously to $\bar{A}$, the section $s$ of $G$ cannot be extended to $\bar{A}$. This implies that the sheaf $G$ of abelian groups on $\bar{A}$ is not flabby. Therefore, $\operatorname{Dim}_{\mathbf{Z}}(\bar{A})>0$, and this completes the proof.

REMARK. Since every countable discrete space is realcompact, Lemmas 2.1 and 2.2 show that if $X$ is locally compact or first countable then, in the proof of the above theorem, we do not have to assume that there does not exist a strongly inaccessible cardinal. In general, however, the proof is based on this assumption.

3. Cohomological dimension and finitistic spaces. In this section we make use of the sum theorem proved in [8] to show that Problem A and a generalization of Alexandroff's problem are equivalent.

It is a theorem of Floyd and Grothendieck (see [4, p. 6]) that if $X$ is locally compact Hausdorff and $L$ is a P.I.D. then the dimension function $\operatorname{dim}_{L}$ agrees with the one defined by Cohen [6]. Further, if $X$ is paracompact Hausdorff as well as 
locally compact then $\operatorname{dim}_{L}$ also agrees with the cohomological dimension defined by Okuyama [13]. The following result relates the covering dimension with these cohomological dimensions with respect to the additive group $\mathbf{Z}$ of integers.

Proposition 3.1. Let $X$ be a paracompact Hausdorff space. Then $D(X, Z) \leqslant$ $\operatorname{dim}_{\mathbf{Z}} X \leqslant \operatorname{dim} X$, where $D(X, \mathbf{Z})$ is the cohomological dimension, $\operatorname{dim}_{\mathbf{z}}(X)$ is the Cartan's cohomological dimension and $\operatorname{dim} X$ stands for the covering dimension. Further, if $X$ is of finite covering dimension then equality holds everywhere.

Proof. First of all we note that for a paracompact Hausdorff space, Čech cohomology and the sheaf cohomology with supports in the family cld of all closed sets are isomorphic. Suppose $D(X, \mathbf{Z})=n$. Then, by definition of cohomological dimension there exists a closed subset $A$ of $X$ such that $H^{n}(X, A ; \mathbf{Z}) \neq 0$. Since $A$ is closed, $H^{n}(X, A ; \mathbf{Z}) \approx H_{\text {cld } \mid(X-A)}^{n}(X-A ; \mathbf{Z})$ where cld $\mid(X-A)$ denotes the family of all closed subsets of $X$ which are contained in $X-A$. Now, because cld $\mid(X-A)$ is a paracompactifying family of supports on the locally paracompact space $X-A$ and its extent equals $X-A$, we find that $\operatorname{dim}_{\mathbf{z}}(X-A) \geqslant n$. Also the subset theorem for $\operatorname{dim}_{\mathbf{z}}$ is true for locally closed subsets of $X$ and hence $\operatorname{dim}_{\mathbf{z}}(X) \geqslant n$. This proves $D(X, \mathbf{Z}) \leqslant \operatorname{dim}_{\mathbf{z}}(X)$ whether or not $X$ is of finite covering dimension. Using Čech's definition of sheaf cohomology, one can easily prove that $\operatorname{dim}_{\mathbf{z}}(X) \leqslant$ $\operatorname{dim}(X)$. Finally, if $X$ is a paracompact Hausdorff space of finite covering dimension, then it has been proved in $[12$, pp. 206, 210] that $D(X, \mathbf{Z})=\operatorname{dim} X$. This completes the proof.

Now we can ask the following

Problem B. Does there exist a paracompact Hausdorff space $X$ of infinite covering dimension such that $\operatorname{dim}_{\mathbf{z}}(X)<\infty$ ?

The universal coefficient theorem for Čech cohomology shows that for a compact Hausdorff space, cohomological dimension with respect to any coefficient group does not exceed the cohomological dimension with respect to the additive group $\mathbf{Z}$ of integers. Hence Alexandroff's problem is equivalent to asking: Does there exist an infinite dimensional compact Hausdorff space which has finite integral cohomological dimension? Therefore, Problem B is a generalization of Alexandroff's problem. Now the following theorem shows that indeed Problems A and B are equivalent.

THEOREM 3.2. The following two statements are equivalent:

(i) Every paracompact Hausdorff space of finite integral cohomological dimension is of finite covering dimension.

(ii) Every paracompact Hausdorff space of finite integral cohomological dimension is finitistic.

Proof. That (i) implies (ii) is obvious. To prove the converse, we shall make use of the following sum theorem: If $\left\{X_{\alpha}\right\}$ is a locally finite closed covering of a paracompact Hausdorff space $X$ and $\operatorname{dim}_{\mathbf{z}}\left(X_{\alpha}\right) \leqslant n$ for each $\alpha$, then $\operatorname{dim}_{\mathbf{z}}(X) \leqslant n$ (cf. [8] for the proof). Now suppose (i) is false and let $X$ be a paracompact Hausdorff space such that covering dimension of $X$ is infinite and integral cohomological dimension is finite, say $n$, for the disjoint sum $Y$ of countably infinite number of copies of $X$. 
Then clearly $Y$ has a locally finite closed covering such that each member of this covering is paracompact Hausdorff and consequently $Y$ is paracompact Hausdorff. Also, by the sum theorem stated above $\operatorname{dim}_{\mathbf{Z}}(Y)=n$. However, one can easily verify that $Y$ is not finitistic. Thus (ii) is false and the converse is proved.

REMARK. Let us observe that a proof of part (ii) of the above theorem will definitely solve Alexandroff's problem in negative, although, disproving it will not quite solve it in affirmative, but may provide a clue to obtain a solution of the above problem either way.

\section{REFERENCES}

1. P. S. Alexandroff, Some old problems in homological dimension theory, Proc. Internat. Sympos. Topology Appl., Herceg-Novi, 1968, pp. 38-42.

2. G. E. Bredon, Sheaf theory, Mc-Graw Hill, New York, 1967.

3. In Introduction to compact transformation groups, Academic Press, New York, 1972.

4. A. Borel, et al., Seminar on transformation groups, Ann. of Math. Studies, No. 46, Princeton Univ. Press, Princeton, N. J., 1960.

5. H. Cartan, Seminar E.N.S., 1950-51.

6. H. Cohen, A cohomological definition of dimension for locally compact spaces, Duke Math. J. 21 (1954), 209-224.

7. S. Deo, Cohomological dimension of a n-manifold is $n+1$, Pacific J. Math. 67 (1976), 155-160.

8. S. Deo and A. S. Shukla, Cohomological dimension and the sum theorems (submitted).

9. S. Deo and H. S. Tripathi, Compact Lie group actions on finitistic spaces, Topology (to appear).

10. L. Gillman and M. Jerison, Rings of continuous functions, Springer-Verlag, New York and Berlin, 1960.

11. Wu-Yi Hsiang, Cohomological theory of topological transformation groups, Springer-Verlag, New York and Berlin, 1975.

12. K. Nagami, Dimension theory, Academic Press, New York, 1972.

13. A. Okuyama, On cohomological dimension of paracompact Hausdorff spaces, Proc. Japan Acad. 38 (1962), 489-494.

14. R. Oliver, A proof of Conner conjecture, Ann. of Math. (2) 103 (1976), 637-644.

15. R. G. Swan, A new method in fixed point theory, Comment. Math. Helv. 34 (1960), 1-16.

Department of Mathematics, University of JAMmU, JAMMU-180001, INDia 